\title{
Fabrication of piezoresistive based pressure sensor via purified and functionalized CNTs/PDMS nanocomposite: toward development of haptic sensors
}

\begin{abstract}
In this work, we reported a chemically modified technique via screen printing method to fabricate carbon nanotubes (CNTs)/Polydimethylsiloxane (PDMS) nanocomposite to monitor the piezoresistive behavior of nanocomposite while applying pressure. Raman, UV/vis and HRTEM results were utilized to verify the quality of CNTs, purified through chemical and physical treatments; carboxylic and hydroxylic functional groups were determined via FTIR. The optimum dispersion ratio of the nanocomposite was observed by FESEM images which clearly show the consistent dispersion of CNTs coated by PDMS. Furthermore, I-V characterization of the developed nanocomposite indicates change in resistivity for a few orders of magnitude before and after treatment in addition to resistance variation as a result of different applied pressures. These results indicate the importance of CNTs treatment prior to nanocomposite fabrication in order to obtain lower percolation threshold. Obtained results are useful in development of haptic sensor, artificial finger, and brain like devices for robotics applications.
\end{abstract}

Keyword: Functional nanotubes; Flexible sensor; Artificial finger; Electrical resistance; Screen printing 